\title{
Top production with D0
}

\section{Viatcheslav Shary*}

SPP, DAPNIA, CEA Saclay, France

E-mail: Viatcheslav.Sharyy@cea.fr

\section{for D0 collaboration}

The measurement of the top quark pair $(t \bar{t})$ production cross-section at hadron colliders can be used to test the standard model and search for new phenomena affecting the top quark sector. We present measurements of the $t \bar{t}$ production cross section at $\sqrt{s}=1.96 \mathrm{TeV}$ in $p \bar{p}$ collisions using $320-370 \mathrm{pb}^{-1}$ of data collected by the D0 experiment during Run II of the Fermilab Tevatron collider.

Within the Standard Model, the top quark almost always decays to a $W$ boson and a $b$ quark. In the dilepton final state both $W$ bosons decay to leptons and neutrinos, so this final state is characterized by the presence of two or more jets, two high transverse momentum isolated charged leptons, either two electrons, one electron and one muon, or two muons, and high missing transverse energy due to the neutrino escaping detection. The combined cross-sections measured in the dilepton final state is:

$$
\sigma_{t \bar{t}}=8.6_{-2.0}^{+2.3}(\text { stat })_{-1.0}^{+1.2}(\text { syst }) \pm 0.6(\text { lumi }) \mathrm{pb} .
$$

In the all-jets final state both $W$ bosons decay to jets and the final state is characterized by the presence of at least six jets, two of them result from the hadronization of $b$ quarks. By using its new silicon tracker, the D0 experiment is capable of identifying $b$ jets based on Secondary Vertex Tagging. Further discrimination between the top signal and the large multijets background is achieved by making use of Neural Network techniques exploiting the differences in kinematic properties of the events. The cross-section measured in the all jets final state is:

$$
\sigma_{t \bar{t}}=5.2_{-2.5}^{+2.6}(\text { stat })_{-1.0}^{+1.5} \text { (syst) } \pm 0.3 \text { (lumi) pb. }
$$

Both measurements are in a good agreement with the standard model prediction of $6.77 \pm 0.42 \mathrm{pb}$.

International Europhysics Conference on High Energy Physics

July 21st - 27th 2005

Lisboa, Portugal

\footnotetext{
* Speaker.
} 


\section{Event Selection}

The top quark is the heaviest fermion and its mass could allow its decay into exotic particles. The inclusive top pair $(t \bar{t})$ production cross section $\left(\sigma_{t \bar{t}}\right)$ can be computed from individual $t \bar{t}$ decay channels and their predicted standard model branching ratios. Exotic top decays would lead to different values of the inclusive top pair production cross section in the different channels. It is therefore important to precisely measure $\sigma_{t \bar{t}}$ in all channels and compare it with the standard model prediction. Within the standard model each top quark of a $t \bar{t}$ pair is expected to decay approximately $99.8 \%$ of the time to a $W$ boson and a $b$ quark [1].

Dilepton final states arise when both $W$ bosons decay leptonically and occur along with two energetic jets resulting from the hadronization of the $b$ quarks and with missing transverse energy from the high transverse momentum $\left(p_{T}\right)$ neutrinos. In this paper we present the D0 [2] measurement using data taken at Fermilab Tevatron collider [3] in the period between April 2002 and August 2004, or 384,368 and $363 p b^{-1}$ in the $e^{+} e^{-}, e^{ \pm} \mu^{\mp}$ and $\mu^{+} \mu^{-}$channels, respectively. The electrons and muons may originate either directly from a $W$ boson or indirectly from a $W \rightarrow \tau \nu$ decay. The corresponding $t \bar{t}$ branching fractions are 1.58\%, 3.16\%, and 1.57\% [1] for the $e^{+} e^{-}$, $e^{ \pm} \mu^{\mp}$ and $\mu^{+} \mu^{-}$channels, respectively. In this final state we select two leptons with $p_{T}>15 \mathrm{GeV}$ and at least two jets with $p_{T}>20 \mathrm{GeV}$. The dilepton final state has a small background mainly from the diboson production $(W W / W Z), Z / \gamma^{\star}$ leptonic decays and the instrumental background where jets are misidentified as leptons. The large missing transverse energy due to the neutrinos escaping detection in the $t \bar{t}$ events is a powerful discriminant against background processes without high $p_{T}$ neutrinos. We apply the missing transverse energy cut in $e^{+} e^{-}, \mu^{+} \mu^{-}$channels with typical value $35 \mathrm{GeV}$ and reject events compatible with $Z \rightarrow e^{+} e^{-}, \mu^{+} \mu^{-}$processes. The sum of the jets and leading lepton transverse momenta as well as sum of two leading eigenvalues of the normalized momentum tensor (sphericity) [4] is used for the additional background suppression. The residual diboson and $\mathrm{Z}$ backgrounds are estimated using Monte Carlo samples generated by ALPGEN followed by PYTHIA. The residual instrumental background is estimated from the data samples enriched with backgrounds events.

The all hadronic final state arises when both $W$ bosons decay hadronically and is characterized by the presence of at least six jets and two of them result from the hadronization of $b$ quarks. This channel has the advantages that all partons from the $t \bar{t}$ process decay to particles that should be visible in the detector (there are no energetic neutrinos produced) and the branching fraction of this state is large (46\% of all $t \bar{t}$ decays). In this channel we select events with at least 6 jets with $p_{T}>15 \mathrm{GeV}$ and pseudo-rapidity $|\eta|<2.5$. Since the cross section of multijet production via the strong interaction is many orders of magnitude larger than the $t \bar{t}$ cross section, multijet background is overwhelming in this channel. We reject the bulk of this background by the requirement of at least one identified $b$-jet in the event, where the Secondary Vertex Algorithm (SVT) is used for $b$-jet identification. The remaining background is rejected by means of a neural network that uses a number of kinematic variables as input. After a cut on the neural network discriminant, a sample enriched in top quark events is obtained. This sample is used to determine the $t \bar{t}$ cross section. We measure the $b$-tagging efficiency in a data sample of dijet events with enhanced heavy flavor content by requiring a jet with an associated muon at high transverse momentum relative to the jet axis. By comparing the SVT and muon-tagged jet samples, the tagging efficiency for semileptonic 


\begin{tabular}{|l|r|r|r|r|}
\hline Category & $e^{+} e^{-}$ & $\mu^{+} \mu^{-}$ & $e^{ \pm} \mu^{\mp}$ & $l^{ \pm} l^{\mp}$ \\
\hline Integrated luminosity $\left(\mathrm{pb}^{-1}\right)$ & 384 & 363 & 368 & \\
\hline \hline$Z / \gamma^{\star}$ & $0.75_{-0.21}^{+0.18}$ & $1.01_{-0.34}^{+0.22}$ & $1.22_{-0.39}^{+0.33}$ & $2.98_{-0.55}^{+0.43}$ \\
$W W / W Z$ & $0.20_{-0.14}^{+0.10}$ & $0.20_{-0.07}^{+0.08}$ & $1.13_{-0.48}^{+0.45}$ & $1.53_{-0.50}^{+0.47}$ \\
Instrumental leptons & $0.09 \pm 0.03$ & $0.13 \pm 0.04$ & $2.13_{-1.66}^{+2.50}$ & $2.35_{-1.67}^{+2.50}$ \\
\hline Total background & $1.0_{-0.3}^{+0.2}$ & $1.3_{-0.4}^{+0.3}$ & $4.5_{-1.8}^{+2.6}$ & $6.8_{-1.8}^{+2.6}$ \\
\hline Signal efficiency & 0.082 & 0.064 & 0.139 & \\
Expected signal & $3.5 \pm 0.4$ & $2.5 \pm 0.3$ & $11.3_{-1.4}^{+1.2}$ & $17.3_{-1.5}^{+1.3}$ \\
\hline \hline SM expectation & $4.5_{-0.5}^{+0.4}$ & $3.8_{-0.5}^{+0.4}$ & $15.8_{-2.3}^{+2.8}$ & $24.1_{-2.4}^{+2.9}$ \\
\hline Selected events & 5 & 2 & 21 & 28 \\
\hline
\end{tabular}

Table 1: Expected background and observed and expected signal yields. The expected signal yield is derived assuming $\sigma_{t \bar{t}}=7 \mathrm{pb}$. The errors on the yields are the quadratic sum of the statistical and the systematic errors.

$b$-quark decays can be inferred. We make use of a Monte Carlo simulation to further correct the measured efficiency to the tagging efficiency for inclusive $b$-quark decays. We estimate the $c$ tagging efficiency from the same simulation, corrected by a scale factor defined as the ratio of the semileptonic $b$-tagging efficiency measured in data to that measured in the simulation. The $t \bar{t}$ event tagging probability is obtained by applying the tagging rates discussed above to each jet in the simulation, taking into consideration its flavor, $p_{T}$, and $\eta$. The average probability to have at least one jet tagged by SVT in a signal $t \bar{t}$ event is $61 \%$.

\section{Results}

In Table 1 we summarize the predicted and observed number of events for the dilepton final state. The $t \bar{t}$ cross section is measured by maximizing the product of the likelihoods for each individual channel based on the Poisson probability to observe a given number of events under the signal-plus-background hypothesis. The preliminary $t \bar{t}$ production cross-sections at $\sqrt{s}=1.96$ $\mathrm{TeV}$ in different dilepton final states at top quark mass $m_{t}=175$ are measured to be:

$$
\begin{aligned}
\text { ee : } & \sigma_{t \bar{t}}=7.9_{-3.8}^{+5.2}(\text { stat })_{-1.0}^{+1.3}(\text { syst }) \pm 0.5(\text { lumi }) \mathrm{pb} . \\
\mathrm{e} \mu: & \sigma_{t \bar{t}}=10.2_{-2.6}^{+3.1}(\text { stat })_{-1.3}^{+1.6}(\text { syst }) \pm 0.7 \text { (lumi) } \mathrm{pb} . \\
\mu \mu: & \sigma_{t \bar{t}}=1.8_{-3.0}^{+4.8}(\text { stat })_{-1.2}^{+1.0}(\text { syst }) \pm 0.1(\text { lumi }) \mathrm{pb} . \\
\text { dilepton : } & \sigma_{t \bar{t}}=8.6_{-2.0}^{+2.3}(\text { stat })_{-1.0}^{+1.2}(\text { syst }) \pm 0.6(\text { lumi }) \mathrm{pb} .
\end{aligned}
$$

The measured cross-section as well as different kinematic distributions are in a good agreement with the standard model prediction of $6.77 \pm 0.42 \mathrm{pb}$ [5] (see e.g. Fig. 1,2). There systematics uncertainty comes from several sources contributing at the same level: jet energy calibration, jet and lepton identification, trigger efficiency.

The $t \bar{t}$ cross-section measured at the all jet final state at top quark mass $m_{t}=175 \mathrm{GeV}$ yields:

$$
\sigma_{t \bar{t}}=5.2_{-2.5}^{+2.6}(\text { stat })_{-1.0}^{+1.5} \text { (syst) } \pm 0.3 \text { (lumi) pb. }
$$

The dominant source of systematics is the uncertainty of the jet energy calibration $(+1.1-0.7 \mathrm{pb})$. 


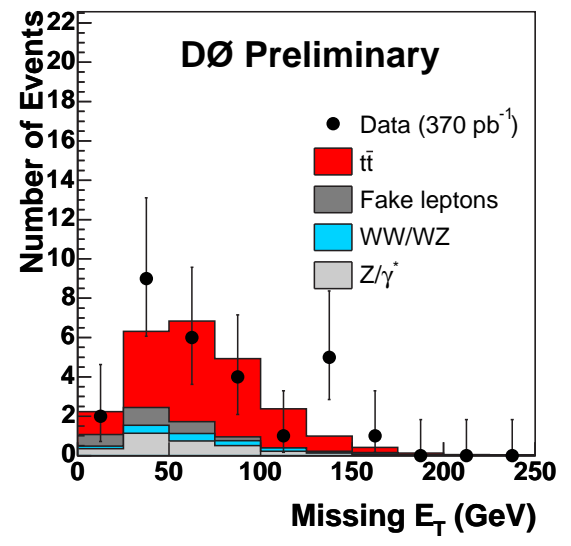

Figure 1: Observed and predicted missing transverse energy distributions in the dilepton final state. The $t \bar{t}$ cross-section assumed to be $7 \mathrm{pb}$.

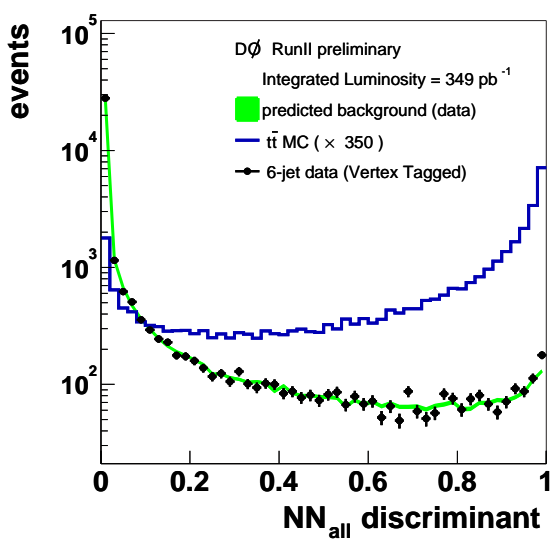

Figure 3: Distribution of $N N_{\text {all }}$ in the all hadronic final state for tagged data (markers), the expected background (grey band) and $t \bar{t}$ (line histogram, multiplied by factor 350$)$. The $N N_{\text {all }}>0.9$ cut is applied for obtaining the sample enriched in $t \bar{t}$ events.

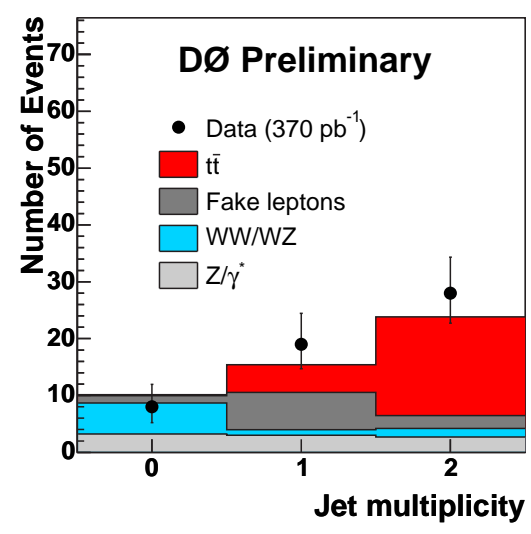

Figure 2: Observed and predicted number of events with 0,1 and 2 or more jets in dilepton final state. The $t \bar{t}$ cross-section assumed to be $7 \mathrm{pb}$.

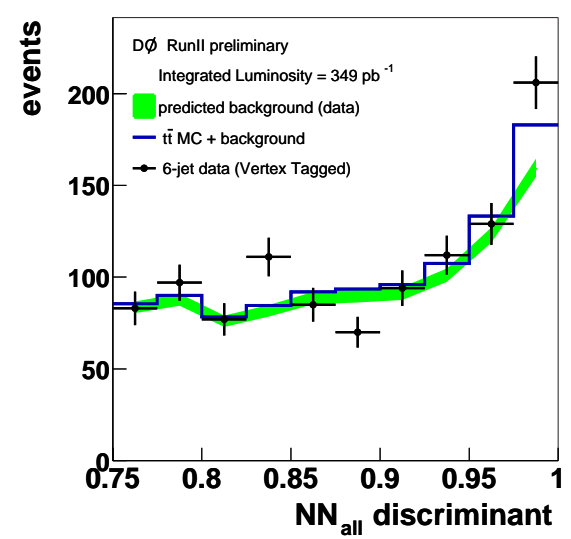

Figure 4: Output of $N N_{\text {all }}$ in the all hadronic final state. Shown is the tagged data (points), the background prediction (band) and the background with $t \bar{t}$ Monte Carlo added to it (histogram, a cross-section of $6.5 \mathrm{pb}$ is assumed).

\section{References}

[1] S. Eidelman et al., Phys. Lett. B 592, 1 (2004).

[2] D0 Collaboration, V. Abazov et al., The Upgraded Do Detector, submitted to Nucl. Instrum. Meth. Phys. Res. A, [hep-physics/0507191; Fermilab-Pub-05/341-E].

[3] CDF Collaboration, D. Acosta et al., Phys. Rev. Lett. 93, 142001 (2004); D0 Collaboration, V. Abazov et al., FERMILABPUB-05-217-E, [hep-ex/0505082].

[4] V. Barger, J. Ohnemus, and R. J. N. Phillips, Phys. Rev. D 48, 3953 (1993).

[5] R. Bonciani et al., Nucl. Phys. B 529, 424 (1998); N. Kidonakis and R. Vogt, Phys. Rev. D 68, 114014 (2003); M. Cacciari et al., JHEP 404, 68 (2004). 